\title{
LA JAZZ BAND Y LA ORQUESTA TÍPICA EN EL FREVO PROCESOS CREATIVOS EN ARREGLOS Y COMPOSICIONES
}

\section{THE JAZZ BAND AND TIPICAL ORCHESTRA IN FREVO CREATIVE PROCESSES IN ARRANGEMENTS AND COMPOSITIONS}

Paulo José de Siqueira Tiné paulotine70@hotmail.com Departamento de Música del Instituto de Artes. Universidad Estatal de Campinas. Brasil

\section{RESUMEN}

El artículo analiza resultados parciales de los últimos proyectos de investigación del autor referidos a los procesos creativos en los arreglos musicales del frevo, género musical del nordeste de Brasil. Estos proyectos están destinados a registrar la producción para un formato idealizado (ensamble brasilero), que entra en tensión con sus antecedentes históricos (orquestas típicas brasileras / jozz bands), ${ }^{1}$ así como con las agrupaciones posteriores llamadas Big Bonds.

\section{PALABRAS CLAVE}

Procesos creativos; arreglo musical; composición musical; música popular; frevo

\section{RESUMO}

0 trabalho discorre sobre resultados parciais obtidos nos recentes projetos de pesquisa sobre processos criativos em música popular do autor dentro do gênero do frevo. Esses projetos tiveram como objetivo registrar a produção para a formação idealizada (ensemble brasileiro), dentro da relação tensional entre ela e antecedentes históricos (orquestras típicas brasileras/Jazz Bonds), bem como com os agrupamentos posteriores chamados de Big Bonds.

\section{PALAVRAS CHAVE}

Processos criativos; arranjo; composiç̃o; música popular; frevo

1 La explicación de la formación de la típica orquesta brosileño se utilizó en otros artículos (Tiné, 2016a). Es una formación inventada para este proyecto que incluye los instrumentos representativos de la música popular de Brasil en una versión compacta que llamé Ensemble Brasileiro. A principios del siglo XX varias agrupaciones fueron llamadas orquestas típicas, aunque no tenían una instrumentación estándar, con el pasar de los años se las denominó jazz bands. El caso del Frevo es un ejemplo característico de este proceso, como en las orquestas de pau-e-corda, que se desarrollará más adelante. 


\section{ABSTRACT}

This article deals with the partial results of the last research projects carried out by the author which make reference to the creative processes in the musical arrangements of frevo, a music genre from the Northeast of Brazil. These projects are aimed to register the production for an idealized format (Brazilian ensemble), which generates tension with its historical antecedents (Typical Brazilian Orchestras/ jozz bonds) as well as with the next groups called Big Bands.

\section{KEYWORDS}

Creative processes; musical arrangement; musical composition; popular music; frevo

La música del nordeste brasilero es una de las más ricas del país. De las manifestaciones rurales a las urbanas hay una serie de géneros, como la Cantoria, el Côco, la Embolada, el Maracatú y el Frevo - entre otros- ligados a folguedos ${ }^{2}$ y manifestaciones de fiestas populares, como el Carnaval y las fiestas de San Juan. De ese modo, el mayor acervo etnomusical de Brasil es el de las Misiones organizadas por Mário de Andrade en el año 1937 que, partiendo de Recife (capital del estado de Pernambuco), adentra el interior de la región para, siete meses después, terminar la expedición en Belém do Pará y registrar en audio, video y fotografía todo lo que dirigía el estudio de los folcloristas de la época.

El frevo es un género carnavalesco nacido en la ciudad de Recife, cuya palabra deriva de «hervir». Así, bandas militares aceleraron la marcha militar, introdujeron ritmos sincopados y los bailarines inventaron el paso, o sea, la danza que acompaña la música. La palabra frevo apareció en los periódicos de Recife en el año 1907, pero las primeras grabaciones fueron realizadas en Río de Janeiro a partir de 1933 por Pixinguinha con el grupo llamado Diabos do Céu, con la clasificación de marcho del norte. En 1949, la antológica Orquestra Tabajara, una Big Bond que luego se dedicó a la ejecución de géneros de la música popular de Brasil, grabó lo que sería una de las principales referencias del repertorio del frevo: Vassourinhas. En la década de 1950 se realizaron las primeras grabaciones en Recife de orquestas recifences, gracias a la discográfica Rozemblit.

El frevo de Pernambuco ha tenido históricamente un proceso interesante en la instrumentación. Inicialmente, los instrumentos de banda marcial marcaban el paso y, poco a poco, algunos instrumentos se fueron incorporando - tales como guitarras y cavaquinhos en el caso de la orquestro de pou e cordo- y otros como tubas y bombardinos se excluyeron hasta que finalmente fueron sustituidos por instrumentos eléctricos, como la guitarra y el bajo eléctrico.

En los grandes clubes [...] el Frevo es tocado por un conjunto musical pesado, con al menos diez instrumentos de metales (pistones, trombones y

2 En Brasil se llama Folguedos a demostraciones populares que reúnen canciones, música, coreografía y temas teatrales. Bien conocido en el país son los bumbo-meu-boi (o boi-bumbá), la Covalhada y las Nau Catorinetos (Cascudo, 2002). 
saxos). La percusión, formada por cajas-sordo, tarol y pandereta, hace el Intermezzo rítmico. [...] el requintista es el músico que más se disputa, debido a la habilidad en las variaciones de improvisación que tendrá que hacer en la ejecución de los frevos [...]. Remanecentes de las grandes bandas marciales continúan practicando sólo sustituyendo el clarinete por la requinta (Rodrigues en Amorim, 2008, p. 18).

En otra parte del libro se aporta la siguiente información:

Se ve en la composición de las primeras fanfarras: clarineta, requinta, dos trombones, dos pistones, dos bajos y un bombardino, para los floreios del contracanto. Por otro lado, en la fanfarria de los «Caiadores», apareció, por la primera vez, el tarol, para sostener mejor el ritmo, función que vino a caber, también, al sordo [...]. Uno y otro nunca más abandonaron las fanfarrias de frevo (Oliveira en Amorim, 2008, p. 28).

Según Maria Alice Amorim (2008), desde la década de los treinta el frevo se dividió en tres subgéneros: el frevo de calle, el frevo canción y el frevo de bloco. Es este último el que aparece en la mencionada orquesta. A pesar de que no había instrumentos de viento en dicho formato, estos terminaron siendo incorporados. En el libro Blocos Carnovalescos do Recife: origens e repertório (1998), se presenta la siguiente instrumentación: flauta, clarinete, dos saxofones alto, dos saxofones tenor, trompeta, trombón/bombardino (barítono), cavaquinho/mandolina/banjo/guitarra (en el mismo pentagrama), contrabajo, percusión (caja/pandereta/surdos).

En esta investigación opté por una agrupación reducida de lo que podría ser una orquesta típica brasilera: dos flautas, dos clarinetes, dos saxofones (un alto y un tenor con posibilidad de usar un soprano), una trompeta, un trombón, dos percusiones, un bandolín, un covoquinho (ukelele brasilero), dos guitarras (una de siete cuerdas), un acordeón y un contrabajo. He partido de la versión reducida del grupo, sin las maderas a dos voces, excepto los saxofones, solamente con una guitarra como representante de las cuerdas y con una batería en lugar de las percusiones. El trabajo se dividió en tres etapas: interpretación de un arreglo característico para dicho formato; confección de un arreglo nuevo para Big Band³ y preparación de una nueva composición para dicho formato.

\section{INTERPRETACIÓN DE UN ARREGLO CARACTERÍSTICO}

Las referencias a Sabe Lá o que é Isso/Hino oos Batutas de São José (1952) se vinculan a la década de 1950. También fue llamado Himno de

3 En este caso, el objetivo es exactamente la relación de tensión o fricción (Piedade, 2011) entre los formatos de Jazz Band y Big Band. 
«los Batutas de San José», compuesto en honor a este grupo de carnaval fundado en 1932, cuya sede se encuentra en el barrio Afogados en Recife. João Santiago dos Reis (1928-1985), que llevó a cabo la Orquesta de Jazz dos Batutas de São José, compuso esta música para el carnaval de 1952. ${ }^{4}$ Sin embargo, el registro más antiguo que se encontró fue realizado por el grupo Quinteto Violado a la colección histórica de discos producidos por Marcus Pereira en la década de 1970, titulado Música Popular do Nordeste. En esa misma década, el sambista Martinho da Vila registró también una interpretación de este frevo. Según la fuente de la partitura utilizada para esta investigación y publicada en 1998 (Blocos Carnovolescos do Recife: origens e repertorio), el arreglo es de Duda de Recife y la transcripción fue realizada por Marco César, aunque sin ninguna indicación de la grabación de referencia.

Uno de los temas que inquietaron al grupo ejecutante de las obras fue cómo interpretar los arreglos escritos a través de la historia. Otro interrogante fue, al tener una interpretación como base, cómo puede esta servir para la confección de un arreglo y de una nueva composición. Aunque este trabajo propone esta dirección, no hay una reproducción exacta de las características dadas. De hecho, se introducen procesos de innovación y elementos ajenos al género. La versión propuesta aquí también realiza una reducción de la disposición original que contiene dos saxofones altos y dos tenores. Por lo tanto, se elige solo la primera de las voces del alto y el tenor.

Para la interpretación grabada en el proyecto del presente artículo se tocó tres veces el arreglo. La primera y la tercera vez cantadas y la segunda con la improvisación del saxofón tenor. Este hecho confirma mi opinión de que los arreglos de frevo en Brasil hacen hincapié en elementos del género como acentos y ritmos, sin variar en las repeticiones y dejan, así, tal posibilidad a cargo de los intérpretes. A pesar de que esta pieza ha sido elegida para ser grabada en esta producción, los grupos que dirigí interpretaban diferentes arreglos de frevo del libro antes mencionado (Silva, 1998) y de otros álbumes de partituras (Capiba \& Moraes, 2007), siempre con estas características musicales.

\section{Duda no Frevo (Senô)}

En este sentido, el arreglo que hice 5 para el clásico Dudo no Frevo, tema compuesto en honor a Duda de Recife, grabado por la Big Band da Santa en el CD del Movimiento Elefontes (Nascimento, 2011), se dirigió exactamente en esa dirección, es decir, la repetición no literal y variación

4 Este punto corrobora con la hipótesis central del estudio de la relación de las antiguas bandas de jazz con los géneros característicos de la música brasileña.

5 Para más información se puede escuchar una grabación del arreglo del frevo Duda no Frevo en el siguiente sitio: https://soundcloud.com/movimentoelefantes/10-duda-no-frevo-big-band-da. 
continua, así como la introducción de la improvisación en el arreglo. Este tema se encuentra en la categoría de frevo de calle, siendo así un frevo instrumental. La instrumentación para Big Band completa parece correcta, aunque este hecho no es necesariamente una regla, puesto que hay numerosos ejemplos de arreglos para formaciones incompletas.

El arreglo está dentro de la forma estándar $A B$ de temas de frevo que operan entre el umbral de una forma binaria o ternaria, ya que ambas secciones están en la misma tonalidad. Este efectúa tres diferentes repeticiones entre la exposición y una vuelta final que tiene función de coda [Tabla 1]:

\section{AA'BB' $\quad$ AA'B $\quad$ B' A $\quad$ A'B $\quad$ B'A Exposición Variación Impro.Sax Impro.Tpt Reexposición Variada Mib Major.........................Sib Major..........................Mib Major}

Tabla 1. Forma del arreglo de Duda no Frevo

El procedimiento de improvisar dentro de la estructura armónica del tema adoptado en la interpretación de Sabe Ló o que é Isso era inusual en el carnaval tradicional. Fue, sobre todo, una de las contribuciones del saxofonista Inaldo Cavalcanti de Albuquerque (1970) y su Spock Frevo Orquestra ${ }^{6}$ que iniciaron ese proceso. Parece claro que deriva de las prácticas de jazz.

El arreglo de Duda no Frevo prevé improvisaciones de saxofón soprano y trompeta que tienen lugar desde la última repetición de la sección B, todavía en la primera variación entrando en la sección A de la segunda variación. La trompeta comienza su improvisación en la repetición (ya en la tercera variación) y se extiende a lo largo de la sección B. Debe tenerse en cuenta que este tema está en la tonalidad mi bemol mayor, pero todas las improvisaciones se producen en tonalidad de si bemol mayor, con el chorus transpuesto, por lo tanto, una quinta arriba. La sección de reexposición vuelve a contar con las melodías de las secciones A y B, pero en este caso, todas rearmonizadas. Por lo tanto, este sería el segundo paso en la presente propuesta, la realización de un arreglo nuevo para una pieza característica de un determinado género.

6 Aquí tenemos una relación tensa, acerca de la identidad brasilera, entre los términos orquesto y Big Bond. Aunque el grupo de Spock es claramente en el formato de Big Bond en una época en que este término ya se había difundido, a diferencia de la antológica Orquesta Tabajara, Spock elige el más viejo término aplicado a Big Bonds en Brasil. 


\section{Bairro de Afogados (2011), de Paulo Tiné}

La inspiración de ese frevo está en el mencionado barrio de la ciudad de Recife que, además de albergar la orquesta Batutas de São José, alojaba también la nación del marocatu Elefante y, por último, en este mismo lugar se encontraba el antológico estudio de grabación Rosemblit, responsable de hacer algunos de los primeros registros del género en la década de 1950 (Telles, 2012) [Figura 1].

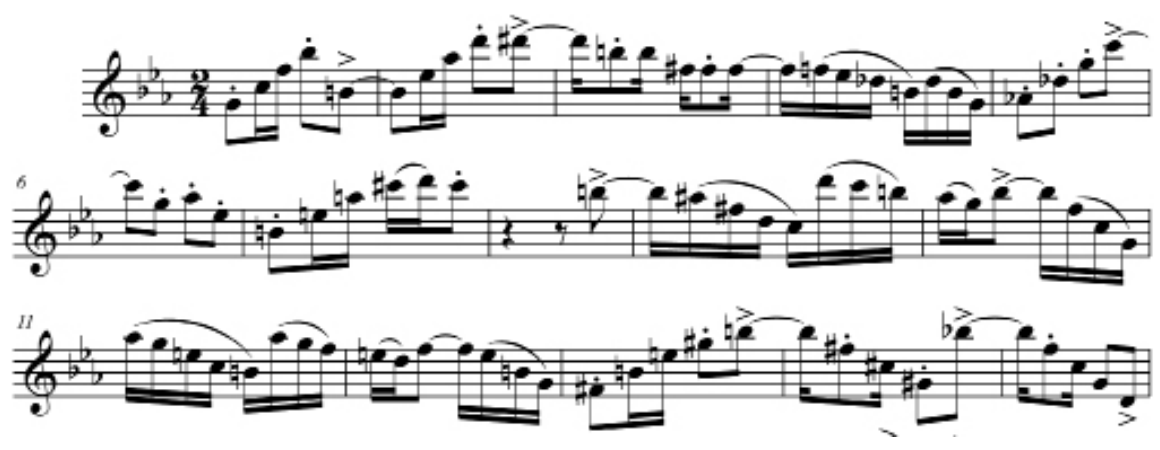

Figura 1. Boirro de Afogodos (2011), de Paulo Tiné. Introducción

Formalmente el arreglo de esta composición se reparte de la siguiente forma:

"Introducción: al unísono /octava entre la Fl. + Bb Cl. + Vio. + Ac. ${ }^{7}$

" Sección A: presentación del tema solamente con la sección ritmo-armónica y la melodía interpretada por el acordeón.

" Sección A': repetición del tema con el conjunto completo, la melodía al unísono entre la guitarra y el saxofón tenor contra una sección ritmo-armónica realizada por el resto del grupo.

" Sección B: contrapunto a tres voces llevadas a cabo mediante la adición de la flauta, saxofón alto y guitarra, más la línea del contrabajo. La cadencia se produce a través de la sección rítmico-armónica.

"Sección A'": melodía ejecutada por el bloque de trompeta, saxofones y flauta (octava arriba), acompañados en puntillismo por otros instrumentos del grupo.

" Sección de improvisación: Esta sección sustituye los cambios armónicos por un solo acorde en la Sección A: B7M(11+); y un solo acorde para la sección B: Csus7(9). Dentro de la forma AABA hay dos cadencias, una que preparan el retorno a la A, y otra para finalizar de la sección.

" Puente: Parte del mismo material de la introducción realizada por el saxofón alto, ahora con la armonía explícita.

7 Las abreviaturas siguen las reglas de la escritura musical. 
* Coda: una invención a dos voces a partir de un tramo de la melodía.

La introducción del tema se basa en la aplicación del coltrane Changes con el género, con una melodía angular interpretado por la flauta y el clarinete añadido a la guitarra y al acordeón.

Además de la estructura armónica hay en el desarrollo melódico el uso de arpegios en cuartas, llamados cuartales, que se moldean a la característica rítmica del género, con el uso de anticipaciones y el llamado garfinho por Hermeto Pascoal o brasileirinho por Mário de Andrade (semicorchea/ corchea/semicorchea). También se destaca que las articulaciones se detaIlan en esta composición. Esto apunta al hecho de que este está impregnado en la escritura musical de la composición. Todavía sin articulación y detalles que conducen a una interpretación más precisa (stoccotos, acentuaciones, crescendos, dinámicas, etcétera), los cuales no se escriben en los arreglos originales del género, de la misma manera que acontece en el Choro, y permanecen en el nivel de la oralidad. Señalé que este hecho se produce también en los arreglos de Pixinguinha (Tiné, 2014b).

La sección A está marcada por arpegios que enfatizan las llamadas tríadas en la camada superior (TCS) o TES, como es nombrado por lan Guest (1996). Por lo tanto, su uso, bastante común e idiomático, está ligeramente desplazado en la medida que los acordes de las triadas no se corresponden directamente con los de la melodía [Figura 2].
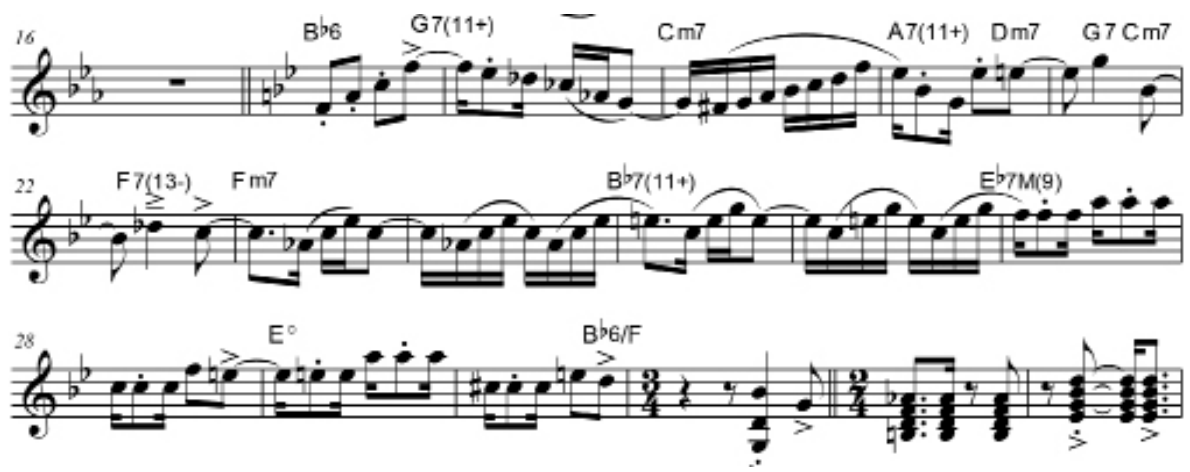

Figura 2. Bairro de Afogados (2011), de Paulo Tiné. Sección A

Se observan tales usos en los siguientes puntos: el arpegio del acorde de F sobre Bb6 (compás 17); Eb sobre A7 (compás 20); Ab en Fm7 (compás 23 y 24); C en Bb7 (25-26); F sobre Eb6 (27-28) y en el E० (29-30). Sumado a esto, la figura de la síncopa característica del frevo (compás 21 y 22) que queda escrita con el uso del tenuto acentuado y el brasileirinho con el punto de stoccoto en la figura central.

Como se ha indicado, la sección B es un contrapunto a tres voces en un total de diez compases. Entonces, hay un retorno a la sección A que suma la 
textura del puntillismo con el bloque de la melodía en tríadas. Este bloque utiliza el mismo TCS o TES mencionadas en la elaboración melódica de la sección. La guitarra lleva a cabo la sección melódica que está fragmentada entre el clarinete y el trombón y esta duplica el contrabajo. El bloque se da solamente en ciertos puntos, tal como muestra la parte del acordeón de la Figura 3 y queda, de esta manera, la secuencia al unísono/ $8^{a}$ hasta entonces. Este hecho es común en los arreglos tradicionales de frevo y armoniza las melodías en los puntos de menor movimiento. La diferencia aquí radica en el hecho de que estas tríadas no son las del acorde fundamental, pero sí de las superposiciones antes mencionadas.

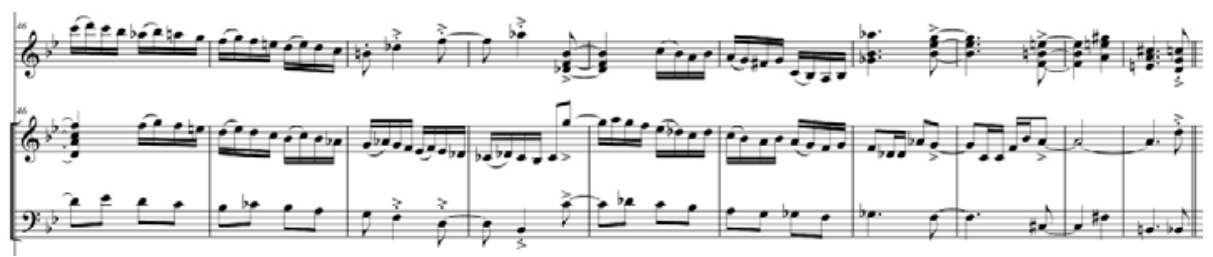

Figura 3. Bairro de Afogodos (2011), de Paulo Tiné. Sección B: acordeón, guitarra e contrabajo

Para la sección de improvisación hice un procedimiento de jazz modal. Este estilo, vinculado al trompetista norteamericano Miles Davis, reemplaza las secuencias tonales, o combios, por acordes estáticos, pero sin modificar la forma. La improvisación se produce dentro de la estructura formal de la composición, pero con la siguiente armonía [Tabla 2]:

\section{A B $\mathbf{A}^{\prime}$ //: Blídio (16c.) :// Cmixolídio (8c.) / C\#m7 F\#7 / Bsus7(9) // Blídio (14c.) / Fsus7(9) Bb6/ \% //}

Tabla 2. Bairro de Afogados (2011), de Paulo Tiné. Improvisación

A pesar de tener algunos lugares con cadencias tonales, como el II - V de $B(-I I)$ y el V de Bb (I), puntuando los cambios de sección, los acordes modales predominan durante la improvisación, si lidio y do mixolidio. Además, la cantidad de compases para cada sección es exactamente la misma de la exposición, es decir, dieciséis con repetición en la sección A, diez en B y dieciséis en la última sección $\mathrm{A}$.

Como se ha mencionado, después de la sección de improvisación realizada por el saxofón tenor, hay un retorno a la melodía de la introducción, aquí, como una especie de puente. También la armonía es construida a 
partir de los Coltrane Changes ${ }^{8}$ del mismo modo que en la introducción, pero entonces estaba implícita, ya que la melodía estaba sin acompañamiento y acá está presente. La Figura 4 muestra la sección a través de los instrumentos mencionados. Sin embargo, la melodía se duplica por los saxofones alto y bajo con el trombón tenor, mientras que la batería marca sólo los contratiempos.

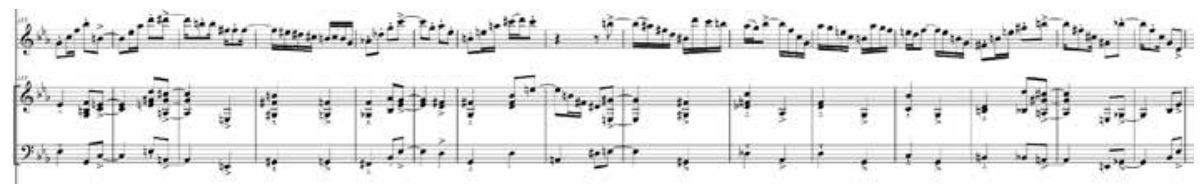

Figura 4. Bairro de Afogados (2011), de Paulo Tiné. Puente: Acordeón, guitarra y contrabajo

Finalmente, la última sección presenta una invención a dos voces hechas a partir de un fragmento del tema [Figura 5]. Sin embargo, hay un proceso de instrumentación que fragmenta la melodía entre los instrumentos y le da la ilusión al oyente de que se trata de más de dos voces, ya que cada nueva entrada suena como una voz más. La guitarra y el acordeón realizan plenamente la invención y están dobladas u octavadas por otros instrumentos del grupo. Al final de este pasaje hay una pequeña coda, con un proceso politonal vinculado a la utilización de las llamadas tríados diatónicas sobre bajos no diatónicos, cuyo efecto muestra insinuaciones de lo que Egberto Gismonti realiza en sus procedimientos vinculados con el compositor Igor Stravinsky en la música brasilera, en las obras como Strowo no Sertão (1996). Tenga en cuenta que, si bien el acordeón realiza la tríada de fa mayor, el bajo lleva a cabo notas fundamentales y quinta justa de sol bemol, mientras que la melodía está en modo de fa mixolidio.

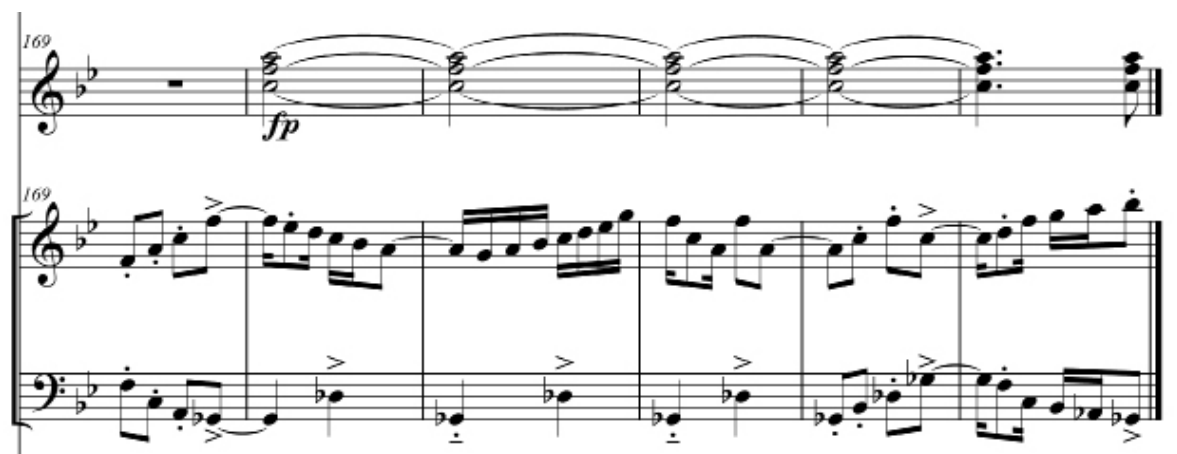

Figura 5. Boirro de Afogados (2011), de Paulo Tiné. Coda final: acordeón, guitarra y contrabajo

8 Se entiende por coltrone changes las incorporaciones de secuencias armónicas simétricas en la cadencia II-V-I realizada por John Coltrane en Tune-up (Miles Davis), a partir de las propiedades exploradas en Giont Steps para la creación del tema Count Down (1960). Para profundizar sobre el tema, se sugiere ver Tiné, 2014. 


\section{CONSIDERACIONES FINALES SOBRE EL FREVO}

El género frevo, así como el samba, también pasó por procesos históricos de adaptación con la batería. La sección de percusión de la partitura de Sabe Ló o que é Isso indica una caja clara, una pandereta (pondeiro) y un surdo. Por lo tanto, la batería puede llevar a cabo la caracterización de este género en el redoblante sumado al bombo de piso, que hace la figura musical del surdo o, en otras ocasiones, realizar la figura de la pandereta (pandeiro) en el plato de conducción. Tales procedimientos serían análogos a los de la samba batucada y samba de plato traducida aquí para este género. Además, hay una posibilidad de realizar la conducción con las escobillas y, por lo tanto, tendría una conducción análoga a la samba escobada. Este último se puede ver en la interpretación de los frevos de Edu Lobo, donde hay una suma del carácter del bossa nova y del género de pernambuco - Ex. «No Cordão da Saideira» (1967) - . Tales procedimientos, en cierto modo, ya forman parte del repertorio de los bateristas, solo que tal vez aún no sean nombrados. Se sabe que, en el linaje de la música instrumental brasilera, el baterista Airto Moreira fue, en gran parte, responsable de la adaptación de los géneros rítmicos de Brasil en el instrumento (Dias, 2013), principalmente con la experiencia del Quarteto Novo. Esto no quiere decir que otras investigaciones no puedan apuntar a otros instrumentistas e, incluso en el contexto de pernambuco, apuntar a otros bateristas importantes en este proceso. ${ }^{9}$ En cualquier caso, dentro de la consignación la llamada música instrumental brasilera, hace que el género, al igual que la samba, usando el plato de conducción en el frevo, sea el modo más adecuado para el momento de la improvisación; hecho que ocurre tanto en el arreglo, como en la composición propuesta.

En cuanto al proceso formal, intenté cambiar deliberadamente el modelo de la repetición literal por la escritura con variación, como es el caso del arreglo de Duda no Frevo que mantuvo la estructura armónica de la música original, aunque con algunos procesos de rearmonización realizados tanto en la sección de improvisación como en la última aparición del tema. En este arreglo, la estructura es siempre la misma y, en ese sentido, se trata de un arreglo estóndor para Big Bond, todavía con procedimientos raros para el género del frevo. Sin embargo, en el caso de la composición Bairro de Afogados, ocurren otros elementos, tales como la sustitución de la armonía original para una sección modal propuesta, aunque estructuralmente no haya ningún cambio. Además, hay una sustitución de la reexposición final por una invención a dos voces y secciones de introducción/puente sumadas a la estructura de tema.

9 Es posible que investigaciones más precisas puedan pesquisar el trabajo del, entonces, baterista Nana Vasconcelos en los grupos en que hay tocado en Pernambuco en los años sesenta, como el Quarteto Yansan y Quarteto Livre, que acompañaban a los artistas como Geraldo Azevedo, Geraldo Vandré, Teca Calazans y Carlos Fernando. 
En resumen, se observa que, por una parte, la propuesta se trasladó a una radicalización de los procesos de Brasil en cuanto a propuesta de instrumentación, repertorio básico y de las características rítmicas. Mientras, por otro lado, los procesos formales y armónicos vinculados a arreglos de jazz y, en algunos casos, con la música clásica, se introdujeron de tal manera que la propuesta sólo puede ser considerada híbrida.

\section{REFERENCIAS}

Amorim, M. A. (2008). 100 anos de Frevo: irreverêncio e tradição. Recife, Brasil: Folha de Pernambuco.

Capiba y Moraes E. (2007). 100 Anos de Frevo [Partitura]. Recife, Brasil: Fundepe.

Cascudo, L. da C. (2002). Dicionário do Folclore Brosileiro. San Pablo, Brasil: Global.

Coltrane, J. (1960). Count Down. En Giont Steps [CD]. Los Ángeles, Estados Unidos: Atlantic Records.

Davis, M. (1954). Tune-Up. En Cookin' with the Miles Dovis Quintet [CD/LP]. Nueva Jersey, Estados Unidos: Prestige.

Dias, G. M. (2013). Airto Moreira: do samba jozz à música dos anos 70 (1964-1975) (Tesis de Maestría). Universidad Estatal de Campinas, Campinas, Brasil.

Estudio Cantareira. (25 de junio de 2013). Bairro dos Afogados (Paulo Tiné) - Ensamble Brasileiro - Barrio de Afogados (Poulo Tiné) [Archivo de video]. Recuperado de https://www.youtube.com/watch?v=|Gu9r-ocb)l

Gaspar, L. (21 de agosto de 2009). Batutas de São José (bloco carnavalesco) [Entrada de blog]. Recuperado de http://basilio.fundaj.gov.br/pesquisaescolar $/$ index.php?option=com_content\&view=articleøid $=495$

Gismonti, E. (1996). Strawa no Sertão. En Meeting Point [CD]. Múnich, Alemania: ECM records.

Guest, I. (1996). Arronjo. Río de Janeiro, Brasil: Lumiar.

Lobo, E. (1967). No Cordão da Saideira. En EDU [CD/LP]. Río de Janeiro, Brasil: Philips.

Nascimento, S. (2011). Duda no Frevo (Arr. Paulo Tiné). Movimiento Elefontes [CD]. Recuperado de

https://soundcloud.com/movimentoelefantes/10-duda-no-frevo-big-band-da.

Piedade, A. (2011). Perseguindo fios da meada: pensamentos sobre hibridismo, musicalidades e tópicas. Per Musi, (23), 103-112.

Silva, L. D. (Org.). (1998). Blocos Cornavalescos do Recife: origens e repertorio. Recife, Brasil: Governo do Estado de Pernambuco.

Telles, J. (2012). Do Frevo oo Monguebeat. São Paulo, Brasil: 34.

Tiné, P. J. de S. (2014a). Harmonía: Fundamentos de Arranjo e Improvisoção. 
San Pablo, Brasil: Rondó.

Tiné, P. J. de S. (2014b). o arranjo de Pixinguinho para Gaúcho de Chiquinho Gonzaga: a Orquestra Típica a caminho da Big Band. Ponencia en el 24. Congresso Nacional da ANPPOM. São Paulo, Brasil.

Tiné, P. J. de S. (2016). Procesos Creativos en arreglos y composiciones del genero Choro para Jazz Band/Orquestra Típica. Vortex, 4(3). 